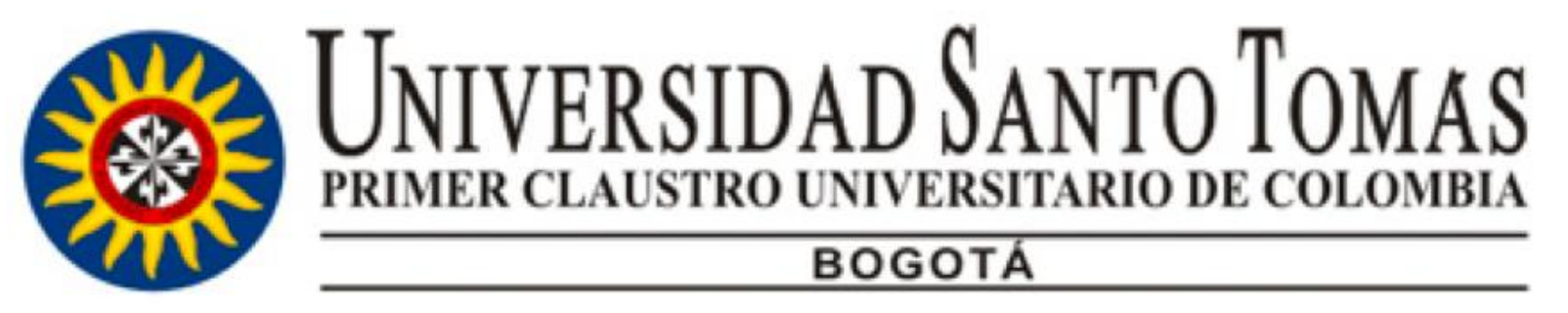

UNIVERSIDAD SANTO TÓMAS

FACULTAD DE ADMINISTRACIÓN DE EMPRESAS

DECISIONES PARA LA DISMINUCIÓN DEL IMPACTO DE LA CRISIS ARGENTINA EN LAS PYMES

AUTORES:

ALVARO DAVID BERMUDEZ SALAMANCA

DANNA GERALDINE MARTINEZ RODRIGUEZ

DAVID EDUARDO NIETO MORA

BOGOTÁ, DICIEMBRE DE 2018 


\section{ÍNDICE}

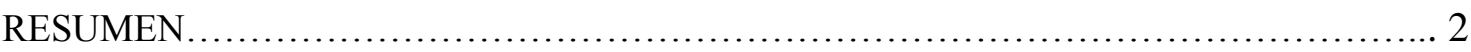

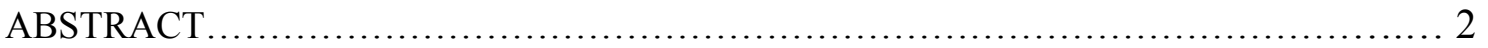

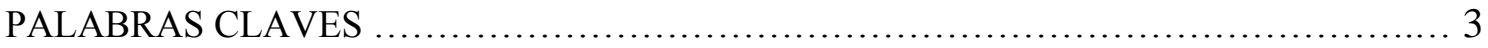

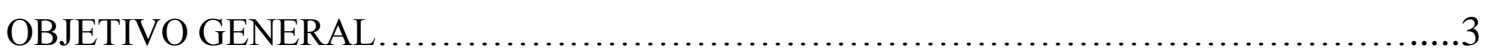

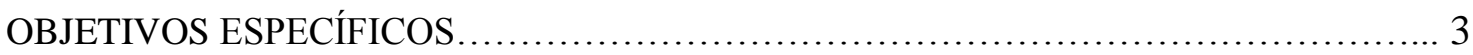

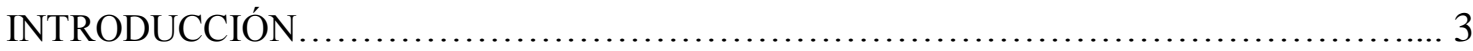

FUNDAMENTACIÓN TEÓRICA.........................................................

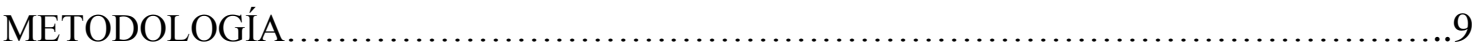

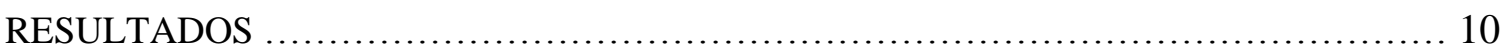

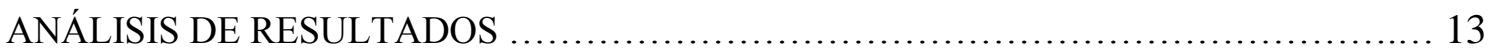

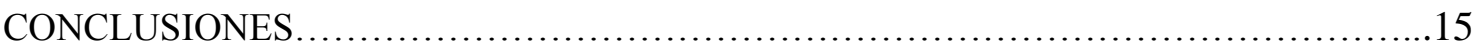

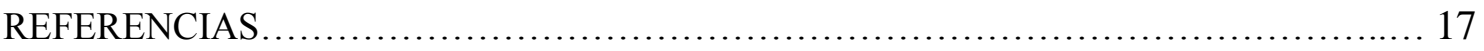

ÍNDICE DE FIGURAS

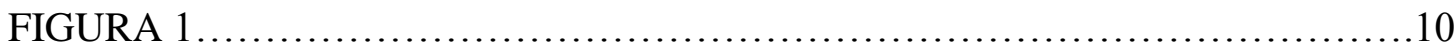

FIGURA 2 ............................................................. 10

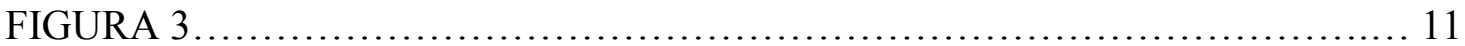

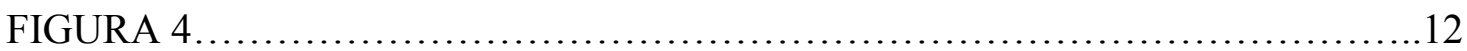

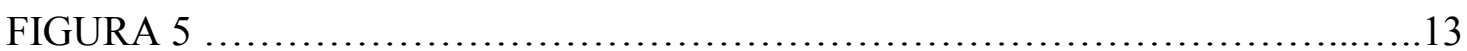




\title{
DECISIONES PARA LA DISMINUCIÓN DEL IMPACTO DE LA CRISIS \\ ARGENTINA EN LAS PYMES
}

\begin{abstract}
Resumen
En el presente trabajo se busca mostrar el impacto generado por la economía argentina en las PYMES, debido a que esta representa cerca del $98 \%$ de las empresas en este país.

Esta investigación ayudará a las PYMES a tomar decisiones en tiempo de crisis así como también poder transformar sus debilidades en fortalezas.

Por otra parte, se debe tener en cuenta que Colombia y Argentina tienen grandes diferencias en varios aspectos; sin embargo, el porcentaje de PYMES que existe en cada economía es alto. Por este motivo se consideró pertinente la realización del presente trabajo desde los dos puntos de vista.

La metodología de esta investigación precisa de una visualización a profundidad debido a que se aportarán estrategias; es por esto que se decidió consultar a personas inmersas en el sector empresarial, todo esto de la mano del conocimiento adquirido en el periplo internacional.
\end{abstract}

\section{Abstract}

This paper seeks to show the impact generated by the Argentine economy in SMEs, because it represents about $98 \%$ of companies in this country.

This research will help SMEs to make decisions in times of crisis as well as to transform their weaknesses into strengths.

On the other hand, it should be taken into account that Colombia and Argentina have large differences in several aspects, however, the percentage of SMEs that exist in each economy is high, for this reason it was considered pertinent to carry out this work from the two points of view.

The methodology of this research requires an in-depth visualization due to the fact that strategies will be provided, that is why it was decided to consult people immersed in the business sector, all this thanks to the knowledge acquired during the international journey 


\section{Tema de investigación}

Decisiones para la disminución del impacto de la crisis Argentina en las PYMES.

\section{Palabras clave}

Economía, crisis, desarrollo, pymes, productividad, creación, calidad.

\section{Objetivo General}

Conocer qué decisiones económicas afectan la creación y sostenibilidad de las PYMES en Argentina

\section{Objetivos específicos}

- Analizar cómo se ven afectadas las PYMES teniendo en cuenta la economía Argentina

- Conocer cómo se encuentra actualmente la economía Argentina.

- Identificar cuáles son los principales factores que afectan la actividad económica de las PYMES.

\section{Introducción}

Desde hace meses se ha venido hablando sobre la inminente crisis económica que ha comenzado a enfrentar Argentina, sus cambios en tasas de interés, su endeudamiento con el FMI, su alta inflación, su baja producción, entre diversos factores que demuestran que el país se encuentra pasando por un momento no muy próspero y positivo para su economía. En consecuencia de esta situación se avecinan diferentes problemas para sus habitantes debido a que su poder adquisitivo disminuye y de ser así las PYMES tendrán menos ingresos, lo que las llevará a grandes endeudamientos que a largo plazo no es lo más recomendable para su sostenibilidad en el tiempo. Lo anterior se debe a que una PYME no tiene un volumen alto de patrimonio y capital que le permita solventar con facilidad estos pasivos y por lo tanto, lo más probable es que no sea competitiva y no se adapte a la situación económica del país. 
Por otra parte, las políticas económicas, establecidas por el último gobierno no han ayudado a solucionar esta crisis. Uno de los retos es disminuir la inflación, que en los últimos meses ha crecido de manera exponencial, saliendo de los pronósticos que tenía el gobierno para el año 2018; donde se esperaba que la tasa de inflación anual llegara al 15\% y al cierre del año se espera que llegue a un 30\%, según los últimos datos de la INDEC (Institutos Nacional de Estadísticas y Censo). Esto ha llevado a que las importaciones tengan unos costos bastantes altos para las empresas, lo que implica una subida de precios a los productos y servicios y en consecuencia las empresas argentinas no sean competitivas y les cueste ser sostenibles en el tiempo.

De acuerdo a lo anterior, la presente investigación pretende conocer cuáles son los principales factores económicos que afectan las PYMES y que determinaciones son necesarias para que su crecimiento y sostenibilidad sean posibles en ejercicio de su actividad económica.

\section{Fundamentación teórica}

En este apartado se busca definir todos aquellos conceptos necesarios para el entendimiento del presente trabajo y de esta forma se logre comprender la finalidad de la investigación.

PYME: "Se consideran pequeñas empresas aquellas que tienen menos de 20 trabajadores y medianas las que tienen entre 20 y 500 empleados. Esta definición es susceptible de variar en función de los distintos contextos económicos e históricos; no existe una definición única que categorice a la pequeña y mediana empresa dado que se utilizan diversos criterios" (Iavarone 2012. p. 10)

Según el Banco Europeo de Inversiones (BEI), se consideran pequeñas y medianas empresas aquellas con menos de 500 trabajadores y con una participación máxima de un tercio del capital en manos de una empresa de grandes dimensiones. 
Las Pymes son un actor fundamental en el desarrollo de un sistema socioeconómico competitivo. Otorgan cambio y competencia al entorno económico; son ellas las que cambian la estructura del mercado. Desde un punto de vista dinámico, la entrada y salida constante de estas empresas del mercado es lo que contribuye a mantener un entorno de competencia, con niveles de precios y rentabilidad que tienden a los de un mercado competitivo. (Paula Iavarone 2012).

Por otra parte y entrando en las debilidades de las PyMEs, se debe tener en cuenta que existen algunas que se presentan con mayor frecuencia debido a el tamaño de la empresa. De acuerdo con Gelmetti, C (2011) una de las principales debilidades es las "Escasas y caras fuentes de financiamiento: Las dificultades financieras de las PYMES han sido una constante para su desenvolvimiento, máxime por las crisis económicas que han debido soportar”, por lo cual se puede concluir que este factor suele aumentarse en tiempos de inflación, dado que las fuentes de financiamiento de las PyMEs aumentan sus tasas de interés como una medida contra la inflación.

Inflación: "Es un proceso económico caracterizado por alzas generalizadas y sostenidas de precios en el tiempo. Por alzas generalizadas de precios se entiende que aumentan todos los precios. Así, los precios de los bienes y servicios, el precio del servicio del trabajo. En otras palabras, sueldos y salarios. En adición, sube también el precio de las monedas extranjeras.” Hugo J. Faría, Carlos A. Sabino, (1997)

La inflación, asimismo, se puede clasificar en cuatro magnitudes, según las clasifica CECREDA $^{1}$ (centro estratégico para el crecimiento y desarrollo Argentino, 2018). Inflación controlada que ronda el 2\%. Inflación moderada que es aquella en la que los precios se elevan gradualmente hasta un $10 \%$. Inflación galopante que es aquella en la que

1 CECREDA: Centro Estratégico para el Crecimiento y Desarrollo Argentino 
los precios suben en tasa de dos o tres dígitos por año, entre $10-20 \%$ y $1000 \%$.

Hiperinflación que refiere a la suba de los precios de hasta 1000\% anual o superiores.

\section{Actualidad de las PYMES en Argentina}

De acuerdo a la resolución 154/2018 del gobierno de Argentina se considera una Pyme a las empresas que en promedio facturen las siguientes cantidades.

Tabla 1

Facturación promedio para ser consideradas PYMES

\begin{tabular}{|c|l|l|l|l|l|}
\hline $\begin{array}{l}\text { Categoría } \\
\text { /Sector }\end{array}$ & Construcción & Servicios & Comercio & $\begin{array}{l}\text { Industria y } \\
\text { minería }\end{array}$ & Agropecuario \\
\hline Micro & $\$ 5.900 .000$ & $\$ 4.600 .000$ & $\$ 15.800 .000$ & $\$ 13.400 .000$ & $\$ 3.800 .000$ \\
\hline Pequeña & $\$ 37.700 .000$ & $\$ 27.600 .000$ & $\$ 95.000 .000$ & $\$ 81.400 .000$ & $\$ 23.900 .000$ \\
\hline $\begin{array}{c}\text { Mediana } \\
\text { Tramo 1 }\end{array}$ & $\$ 301.900 .000$ & $\$ 230.300 .00$ & $\$ 798.000 .000$ & $\$ 661.200 .000$ & $\$ 182.400 .00$ \\
\hline $\begin{array}{c}\text { Mediana } \\
\text { Tramo 2 }\end{array}$ & $\$ 452.800 .000$ & $\$ 328.900 .00$ & $\$ 1.140 .300 .000$ & $\$ 966.300 .000$ & $\$ 289.300 .000$ \\
\hline
\end{tabular}

Fuente: Ministerio de producción y trabajo de la Argentina. Resolución (SEyPyME) $154 / 2018$

Por otra parte, de acuerdo con el Ministerio de producción de Argentina existen alrededor de 853.886 PYMES de las cuales solo el 42\% (358.505) se encuentran registradas en AFIP ${ }^{2}$, y de estas, el $46.1 \%$ se encuentran en el sector de industria, el $66.8 \%$ en el sector agropecuario, el 35,8\% en el sector de comercio, el 39,7\% en el sector de servicio, y el $37,7 \%$ en el sector de la construcción.

Según los últimos datos brindados por el GPS de empresas del Ministerio de Producción en el país existen alrededor de 856 mil empresas registradas, de las cuales 606.000 son empleadoras. El 99,4\% (602.784) tiene menos de 200 ocupados, y sólo el 0,6\% (3.532) son

\footnotetext{
${ }^{2}$ AFIP: Administración Federal de Ingresos Públicos
} 
grandes empresas con más de 200 ocupados. Las empresas de hasta 200 empleados son las que más trabajadores emplean: cuentan con el $65 \%$ del empleo privado formal.

Utilizando la misma fuente de datos, se puede afirmar que las MiPymes representan un $64,7 \%$ del empleo privado registrado. Las microempresas son responsables del 21\%; las pequeñas empresas del 22,5\%; y las medianas de 21,2\%. Informe Cámara de comercio Argentina (Julio, 2018)

Desde el punto de vista de las obligaciones, las PYMES en Argentina tienen una de las cargas tributarias más altas del mundo, como se observa en la siguiente tabla.

Tabla 2

Cargas tributarias

\begin{tabular}{|l|l|}
\hline \multicolumn{1}{|c|}{ País } & $\begin{array}{l}\text { Tasa de impuesto total } \\
\text { (\% de ganancias) }\end{array}$ \\
\hline Argentina & 106 \\
\hline Bolivia & 83,7 \\
\hline Eritrea & 83,7 \\
\hline Guinea Ecuatorial & 79,4 \\
\hline Palau & 75,4 \\
\hline República \\
Centroafricana & 73,3 \\
\hline Mauritania & 71,3 \\
\hline Colombia & 69,8 \\
\hline Brasil & 69 \\
\hline China & 68,5 \\
\hline
\end{tabular}

Fuente Los 10 países que más y menos impuestos cobran. Recuperado de http://www.http://diarioretailsudamericabusiness.com

De tal manera las políticas y decisiones del estado argentino hacen que sea difícil la generación de ganancias por parte de las PyMes y la sostenibilidad en el tiempo. Lo cual a su vez no es un incentivo para la inversión extranjera. 


\section{Crisis argentina}

Argentina, uno de los países líderes en economía dado que tiene un alto porcentaje de PIB pero con un índice de crecimiento negativo ha venido sufriendo una alta inflación y un déficit fiscal "En casi tres años de mandato, esta es quizá la crisis más grave que ha enfrentado el presidente, Mauricio Macri, cuyo gabinete anunció este jueves dos medidas para contener la devaluación: vender US\$330 millones en el mercado de valores y subir la tasa de interés al 60\%, la más alta del mundo”. (Daniel Pardo, 2018) Ahora bien, se debe tener en cuenta que "El peso cayó un 13,1 por ciento, a 39,25 unidades por dólar, pese al anuncio previo del jefe de Gabinete, Marcos Peña, de que el Gobierno acelerará una reducción del déficit fiscal para traer tranquilidad a un mercado que desde mayo ha estado bajo presión.”(Reuters, 2018). Esta devaluación del peso trae consigo diferentes barreras de comercio y expansión que impiden que las PYMES tengan un crecimiento; esto, debido a que sus costos aumentaron y las tasas de interés serán altas, por lo tanto, las utilidades necesarias para el funcionamiento de cualquier empresa no solventaran las deudas que habitualmente tiene las PYMES.

La crisis actual de Argentina, es una situación que se venía venir, desde el gobierno de Cristina Kirchner. ¿Qué sucede hoy? A Argentina le faltan dólares. La inflación galopante devoró la ventaja comparativa del tipo de cambio "alto". Con costos de salarios en dólares que aumentan un $20 \%$ cada año, las empresas pierden competitividad. El boom del consumo se sostiene cada vez más con las importaciones. Ante la pérdida del valor de la moneda, los ahorristas huyen hacia el dólar; buscan ponerse a salvo de una devaluación. Carlo Pagni (2012).

\section{Metodología}


Este informe tiene como objetivo analizar e identificar los factores y decisiones económicas que afectan la creación y sostenibilidad de las PYMES en la Argentina; del mismo modo se busca dar una claridad acerca del entorno en el que se desenvuelven estas.

Para ello esta investigación está basada en fuentes primarias y secundarias, tales como, fuentes bibliográficas, elementos fotográficos, y experiencias aportadas en cada una de las visitas académicas en Argentina, así como también gerentes de las PYMES en Colombia a los cuales se les aplicará una encuesta la cual dará la posibilidad de realizar una comparación entre las PYMES colombianas y argentinas y de esta manera poder conocer cuáles son los principales factores que afectan la actividad económica de las PyMes.

En referencia a la actual situación económica argentina y el impacto que esta tiene en el desarrollo de las PYMES, se desean identificar los factores que afectan el crecimiento organizacional y sostenibilidad en el tiempo.

Se realizará una investigación de tipo descriptivo analítico que de acuerdo a Niño, V (2011) una investigación descriptiva tiene como propósito “describir la realidad objeto de estudio, un aspecto de ella, sus partes, sus clases, sus categorías o las relaciones que se pueden establecer entre varios objetos, con el fin de esclarecer una verdad, corroborar un enunciado o comprobar una hipótesis". Para esto se realizará un cuestionario que de acuerdo a Hernández, Fernández, \& Baptista (2010) “Tal vez sea el instrumento más utilizado para recolectar los datos, consiste en un conjunto de preguntas respecto de una o más variables a medir.” es por eso que para la presente investigación se hará uso de este instrumento.

Se considera una investigación no experimental con un diseño transversal; es decir, los datos se recolectarán en un momento único, en este caso en el mes de Noviembre del año 2018.

\section{Población}


Para la ejecución del presente trabajo se realizó una observación a las organizaciones visitadas en Argentina en el mes de noviembre, más exactamente entre los días 10 a 17. Adicionalmente, para tener una visión comparativa se escogerá a directivos en las PYMES colombianas a los cuales se les aplicará un cuestionario, los participantes deberán cumplir con los siguientes criterios:

\section{Criterios de inclusión (Cuestionario aplicado en Colombia)}

- Pertenezcan a una PYME colombiana

- Mayores de 22 años

- Ubicados en la ciudad de Bogotá

- Que pertenezca al área administrativa

- Que pertenezcan hace más de 3 años a la organización

\section{Resultados}

En primera medida se darán a conocer los resultados de la encuesta, para continuar con un análisis de los resultados que permita ligarlo a los objetivos buscados.

En los factores demográficos de los encuestados, la edad de las personas se encuentra entre los 22 a los 59 años y el $60 \%$ de ellos son hombres mientras que un $40 \%$ son mujeres.

\section{Genero}
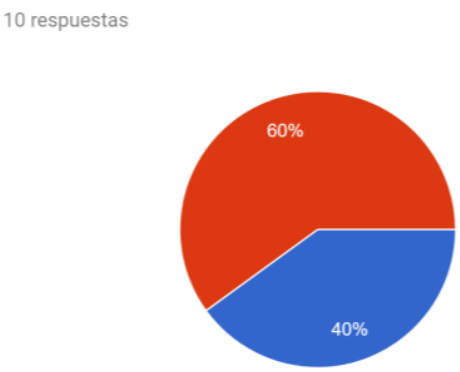

Figura 1, Género

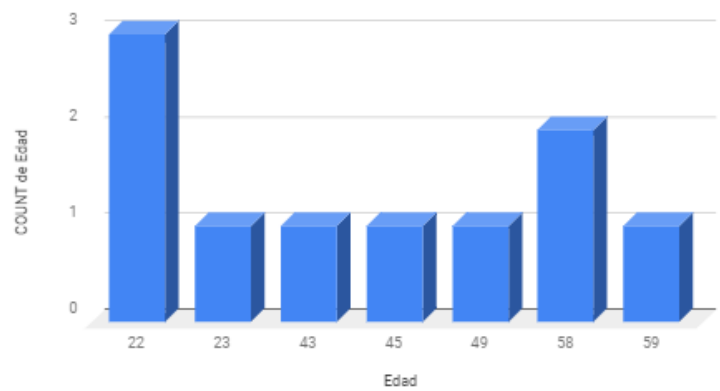

Figura 2, Edad 
Para conocer cuáles eran las alternativas que le ayudarán más a las PyMes argentinas se establecieron distintas opciones en las cuales los encuestados debían escoger entre 1 y 5 siendo 5 muy viable y 1 no tan viable; para el desarrollo del presente ejercicio solo se presentarán aquellas alternativas que tuvieron mayor calificación de ser viables, sin embargo, si se desean conocer las demás respuestas se encontrarán en el Anexo 1.

Una de las alternativas que mayor calificación tuvo fue "Hacer lo posible por disminuir los costos de la empresa"

5. Hacer lo posible por disminuir los costos de la empresa 10 respuestas

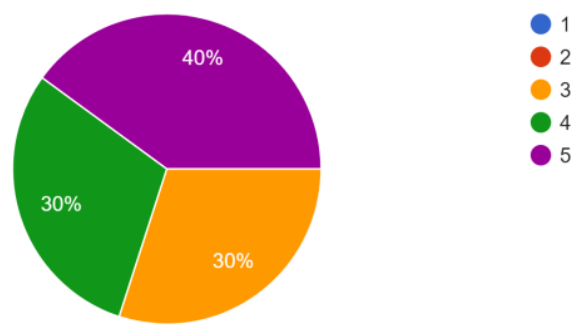

Figura 3, Disminuir costos

Con un 40\% de votación es la opción más viable para disminuir el impacto de la inflación en las PyMes; los encuestados consideran que entre las opciones ofrecidas el disminuir los costos les ayudará mucho en los distintos impactos negativos que traen consigo una inflación. Sin embargo, se debe tener en cuenta que disminuir costos no implica disminuir la calidad de los productos, esto se concluye debido a que en la segunda opción mejor calificada por los encuestados fue "Aumentar o mantener la calidad de sus productos para volverlos más competitivos" 

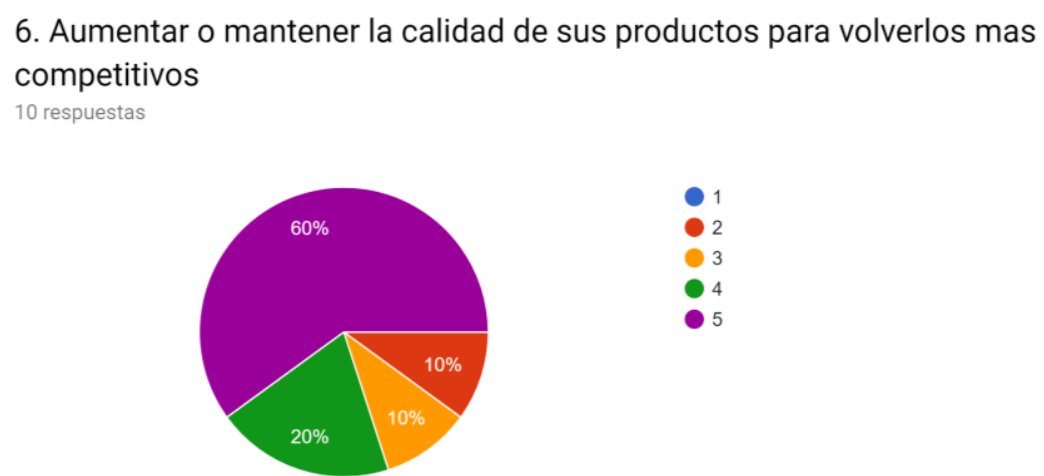

Figura 4, Calidad de los productos

En esta opción un $60 \%$ de los encuestados contestaron que la opción de aumentar y mantener la calidad de sus productos para volverlos más competitivos es una de las decisiones más viables que disminuiría el impacto de la inflación en las PyMes. Esta opción afirma lo observado en la empresa argentina llamada Súper Bol, pues en la experiencia que se logró vivir, el creador de la empresa afirmaba que lo que había permitido que Súper Bol se mantuviera vigente luego de tantas crisis vividas, es el ofrecer los productos con calidad, lo que lleva a que sus clientes se fidelicen con su empresa y por lo tanto en los momentos de crisis no se vean con la necesidad de disminuir su calidad y precios para atraer más clientes.

Por otra parte, se cuestionó a los encuestados si consideran que la carga tributaria afecta a las PyMes en Colombia, debido a que en Súper Bol también se afirmaba que la carga tributaria que ellos tienen es bastante alta, lo que los ha llevado a dejar de lado proyectos como por ejemplo la certificación de calidad, por cumplir sus deberes con el gobierno.

En esta pregunta un $70 \%$ de los encuestados consideraron que la carga tributaria afecta mucho a la PyMes en Colombia; es decir que este factor también es uno de los que más afectan no solo a las PyMes argentinas sino también a las PyMes Colombianas. 
1. ¿Cuanto considera usted que afecta la carga tributaria a las PYMES en Colombia?

10 respuestas

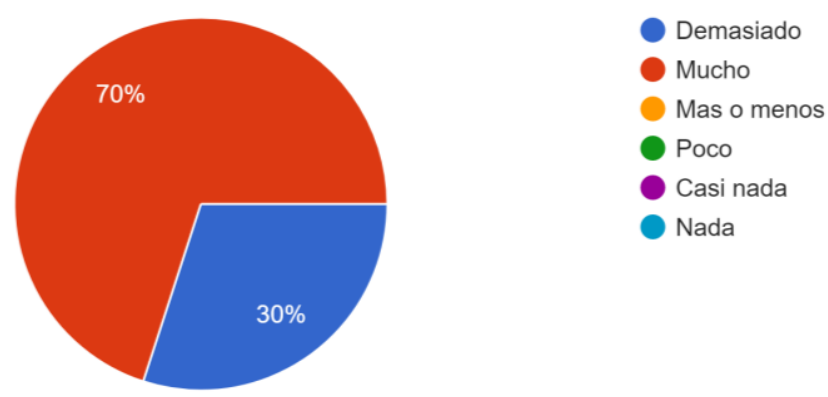

Figura 5, Carga Tributaria

\section{Análisis de resultados}

Para el desarrollo del análisis de resultados del presente trabajo se tendrá en cuenta la 5 edición "Encuesta a PYMES de PwC Argentina, Expectativas 2018 Como se preparan las PYMES para los nuevos desafíos", la cual es una encuesta que se realiza cada año a los empresarios argentinos, que constituyen pequeñas y medianas empresas. Este estudio muestra datos reales, de cómo ven los empresarios la situación económica del país, para las PYMES.

Para los empresarios argentinos, la situación que más les preocupa es la carga tributaria. Como ya se conoce, Argentina, es el país con los impuestos más altos de Latinoamérica; por lo tanto, esta medida del estado, no impulsa la creación de PyMES y aleja a la inversión extranjera. Esto no es un dato menor, debido a que a las PyMES, les cuesta ser sostenibles y generar utilidades, para reinvertir.

Ahora bien, cuando se pregunta a los empresarios: ¿Cuáles son los temas que más les preocupa? El 80\% considera que la presión tributaria. PwC Argentina (2018). La situación en Colombia no es muy lejana de Argentina, ya que Colombia también es uno de los países con carga tributaria más alta en Latinoamérica. 
Teniendo en cuenta lo anterior se puede afirmar que este estudio realizado en Argentina se asemeja a las respuestas obtenidas en la encuesta aplicada en Colombia, donde los empresarios también consideraban que se les aplicaban demasiados impuestos; esto lleva a concluir que una amenaza como esta se debe enfrentar y transformar; puede ser a través de convenios que permitan la reducción de impuestos por reciclaje, por contratación de personas en condición de discapacidad o por donaciones realizadas a entidades sin ánimo de lucro y como beneficio de esto generar una responsabilidad social empresarial.

Por otro lado, la inflación de Argentina en el 2018, es de un 30\% anual, siendo la segunda más alta de Latinoamérica, Diario EL PAÍS (Julio 2018), lo cual la ubica como la segunda preocupación de los empresarios argentinos con un $68 \%$, y no es para menos. Debido a que a las PyMES les afecta en toda su cadena productiva, y en consecuencia lo más probable es que los costos de los insumos de importación suban y a su vez, los salarios de los trabajadores deban aumentarse también. Lo cual no es una situación favorable, para las PYMES, debido que los costos de producción suben y esto genera que el margen de utilidad sea menor. Y sin olvidar la carga tributaria, que se suma a estos factores que son los principales causales de que muchas PyMes en la Argentina desaparezcan en los últimos años.

Otra situación que no ha favorecido a las PYMES, es la tasa de interés que maneja el Banco de la República Argentina, la cual se incrementó a un 60\%, siendo la más alta del mundo. Diario Portafolio (Agosto 2018) El financiamiento de emprendimientos o nuevos proyectos, es casi inmanejable y no solo para los empresarios, sino para la gente del común, se sabe que con una tasa tan alta, no se incentiva el consumo y no es saludable para la economía del país.

A pesar de la situación, los empresarios argentinos, consideran que las acciones para afrontar la crisis o los nuevos desafíos son; desarrollo de nuevos negocios con un $61 \%$, reducción de costos con un $42 \%$, inversión en marketing digital con un $42 \%$. De acuerdo a 
PwC Argentina (2018). Al igual que los empresarios colombianos, buscan reducir costos de producción y ser más competitivos.

En la encuesta realizada en Colombia se realizó una pregunta en la cual se buscaba conocer una opinión abierta y libre del empresario colombiano. En esta se le preguntaba qué le aconsejaba a un empresario argentino desde su punto de vista; de las 10 respuestas obtenidas en 6 se mencionó la palabra calidad, es decir que aun teniendo la posibilidad de escribir cualquier respuesta, los conocedores del sector empresarial continúan considerando que la calidad en esencial, así como también lo permitieron conocer los empresarios argentinos en la experiencia vivida. Por ejemplo, la empresa Campari dedicada a la producción de aperitivos afirmaba que trabajaban en ofrecerles la mejor calidad a los clientes y al realizar sus campañas de complementación del aperitivo con espumantes, lo hacían con los de más alta calidad para mantener la experiencia que ellos querían ofrecer con su campaña. Del mismo modo contrataban a los más expertos bartenders de todas las partes del mundo para seguir con la línea de calidad de la experiencia.

Continuando con las experiencias que permitió vivir la misión académica, los funcionarios y directivos de la empresa Súper Bol (la cual fue la que inspiró la realización del presente trabajo), hablaban de las diferentes certificaciones que ellos tienen en la producción de sus bolsas y aunque no han logrado conseguir una certificación deseada debido a la crisis de Argentina, ellos continúan trabajando bajo las políticas de calidad que exige esta certificación con el fin de ofrecerle lo mejor a sus clientes.

\section{Conclusiones}

- El escenario para las PYMES en Argentina, no es el mejor, debido a su alta inflación y carga tributaria, lo cual disminuye las oportunidades de las PyMES. Estas se 
encuentran en un nivel en el que pueden innovar y generar un valor agregado, buscando ser empresas competitivas, no solo en mercado nacional, sino internacional.

- Antes de considerar cualquier opción para sobrellevar la inflación es importante pensar en la calidad que se le está ofreciendo al consumidor; a partir de este aspecto se puede llegar a diferenciar la empresa y por lo tanto el cliente estará dispuesto a pagar un precio mayor por un producto de mayor calidad.

- La principal preocupación de las PyMes en Colombia y en Argentina es la alta carga tributaria que se les impone; por lo tanto, es una barrera que impide que estas empresas tengan un crecimiento constante y que puedan destinar su margen de rentabilidad a otras prioridades que le podrían ayudar a mejorar su servicio / producto.

- Tener una estrategia dirigida a volumen de ventas no es una de las mejores decisiones, dado que esto implica disminuir precio para que aumenten la cantidad de ventas. Esta estrategia podría tener distintas interpretaciones por parte del cliente como por ejemplo mala calidad, no diferenciación del producto, y ya que son clientes atraídos por precio probablemente así mismo se irán cuando se deban aumentar estos.

\section{REFERENCIAS}

- Cámara argentina de comercio. (2018). LAS PYMES: ANTÍDOTO CONTRA LA POBREZA. Noviembre 21, 2018 , de Cámara argentina de comercio y servicios Sitio web: http://www.cac.com.ar/data/documentos/55_Documento\%20CAC\%20\%20Pyme\%202018.pdf

- Gelmetti, C. J. (2011). Pymes globales: estrategias y prácticas para la internacionalización de pymes (2a. ed.). Retrieved from https://ebookcentral.proquest.com

- Hernández; Fernández \& Baptista, P... (2010). Metodología de la Investigación. México: Mc Graw Hill. 
- Ibáñez, J. (2018). LOS 10 PAÍSES QUE MÁS Y MENOS IMPUESTOS COBRAN. Noviembre 26, 2018 , de Sudamérica Business Sitio web: http://diarioretailsudamericabusiness.com/usa/4927/

- Iavarone, P... (2012). OSTOS POR ÓRDENES DE PRODUCCIÓN: SU APLICACIÓN A LA INDUSTRIA PANIFICADORA. Noviembre 20, 2018, de Universidad nacional de Cuyo Sitio web: http://bdigital.uncu.edu.ar/objetos_digitales/5230/iavaronitrabajodeinvestigacion.pdf

- Ministerio de producción. (2018). PyMEs Registradas. Noviembre 21, 2018, de Ministerio de producción Presidencia de la nación Sitio web: https://www.produccion.gob.ar/pymesregistradas/

- Niño, V. (2011). Metodología de la investigación. Bogotá: Ediciones de la U - PAGNI, C. (2012). LA CRISIS ARGENTINA: El experimento populista de los Kirchner. Cuadernos De Pensamiento Político, (35), 97-112. Retrieved from http://www.jstor.org/stable/23265791

- Pardo, D. (2018). 3 claves que explican qué está pasando con la economía en Argentina (y cómo puede afectar al resto de América Latina). Noviembre 21, 2018 , de BBC Sitio web: https://www.bbc.com/mundo/noticias-america-latina-45361764

- Pwc. (2018). Expectativas 2018 Cómo se preparan las Pymes para los nuevos desafíos. Noviembre 26, 2018 , de Pwc Sitio web: https://www.pwc.com.ar/es/publicaciones/assets/expectativas-2018.pdf

- Resolución (SEyPyME) 154/2018, Micro, pequeñas y medianas empresas. Se actualizan los parámetros y requisitos para solicitar beneficios a partir del 10/5/2018. República de Argentina.

- Diario EL PAÍS. (2018). La inflación argentina supera en el primer semestre el pronóstico para todo 2018. Julio 17, 2018, recuperado El PAÍS sitio web: 
https://elpais.com/economia/2018/07/17/actualidad/1531858015_590345.html.

- Portafolio. (2018). Argentina sube su tasa de interés al 60\%. Agosto 30, 2018, recuperado Portafolio Sitio web:https://www.portafolio.co/internacional/argentinasube-su-tasa-de-interés-520623

- Jonatán Clara, Cristian Pettico, Carolina Suárez, Diego Martín Cuneo, (2018) . "INFLACIÓN EN LA ARGENTINA “Ciclos inflacionarios en Argentina, definiciones, y teorías, de CECREDA sitio web: http://www.cecreda.org.ar/archivos/INFORME_08_01_15.pdf 


\section{DIA 1, DOMINGO 11 NOV 2018}

\section{CITY TOUR POR BUENO AIRES}

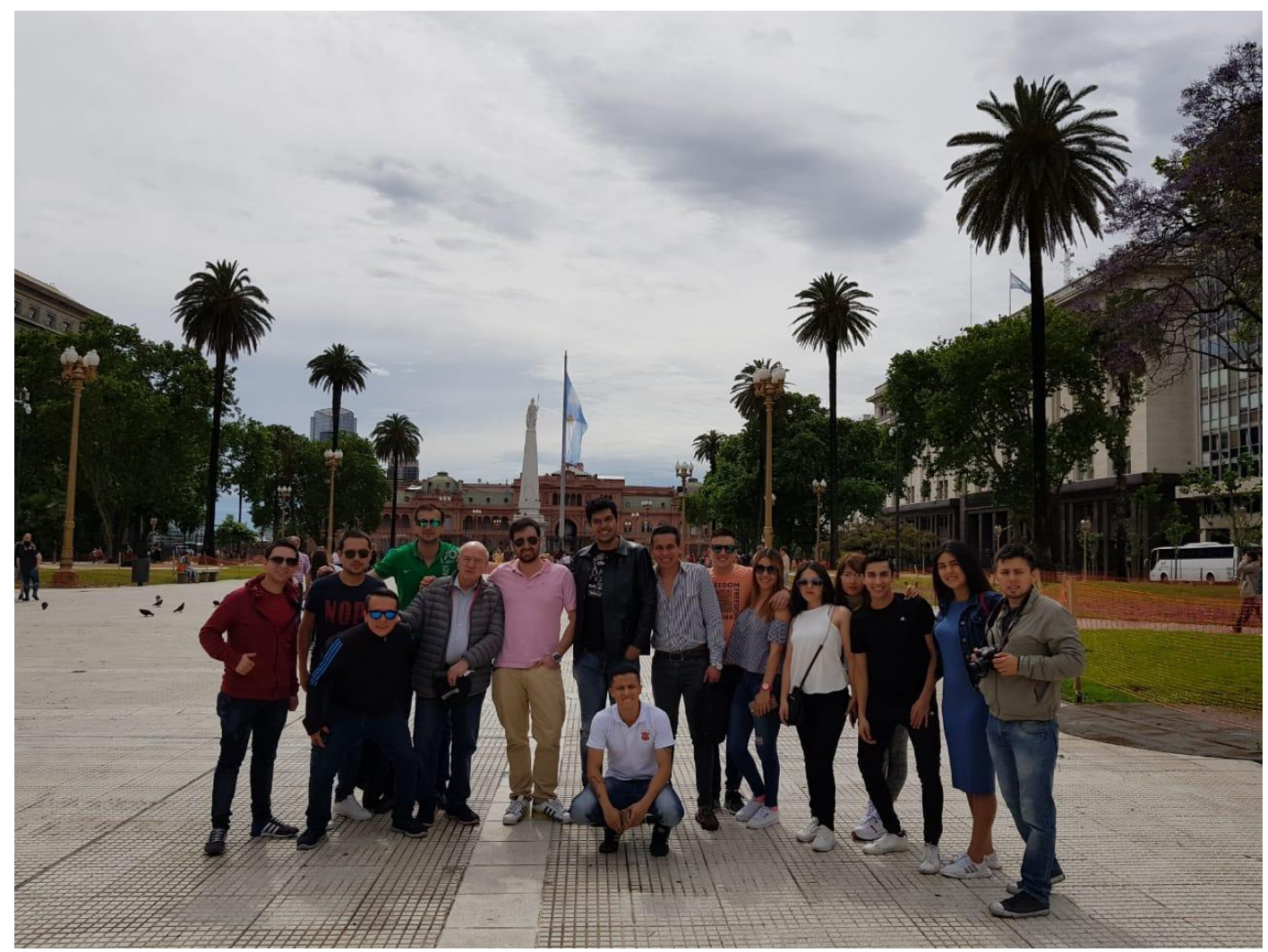

El primer día de esta misión académica, se destinó para conocer Buenos aires, recorrimos sus calles principales, fuimos a la casa rosada, al caminito y luego de esto nos dirigimos al restaurante, donde pudimos probar algunos de los platos típicos de Argentina. Todos nos encontrábamos agotados, ya que el día anterior estuvimos esperando por bastante tiempo el vuelo, debido a que lo retrasaron varias veces, por eso algunos optaron por ir a descansar al hotel, mientras que otros fueron a un barrio llamado Palermo, allí nos reunimos para disfrutar de la cultura futbolera de Argentina, donde pudimos presenciar la ida de la final de la copa libertadores entre Boca Juniors y River Plate. Clubes más representativos de Argentina y oriundos de la ciudad de Buenos Aires, en la historia del fútbol jamás se había vivido un evento de tal magnitud y ante la rivalidad de estos dos clubes la ciudad estuvo paralizada y los protocolos de seguridad por parte de la policía 
nacional no se hicieron esperar, se vivió un ambiente futbolero, nunca antes vivido que para nuestra fortuna terminó muy bien y pudimos regresar al hotel a descansar.

\section{DIA 2, LUNES 12 NOV 2018}

\section{VISITA SUPER BOL Y VISITA BOMBONERA}

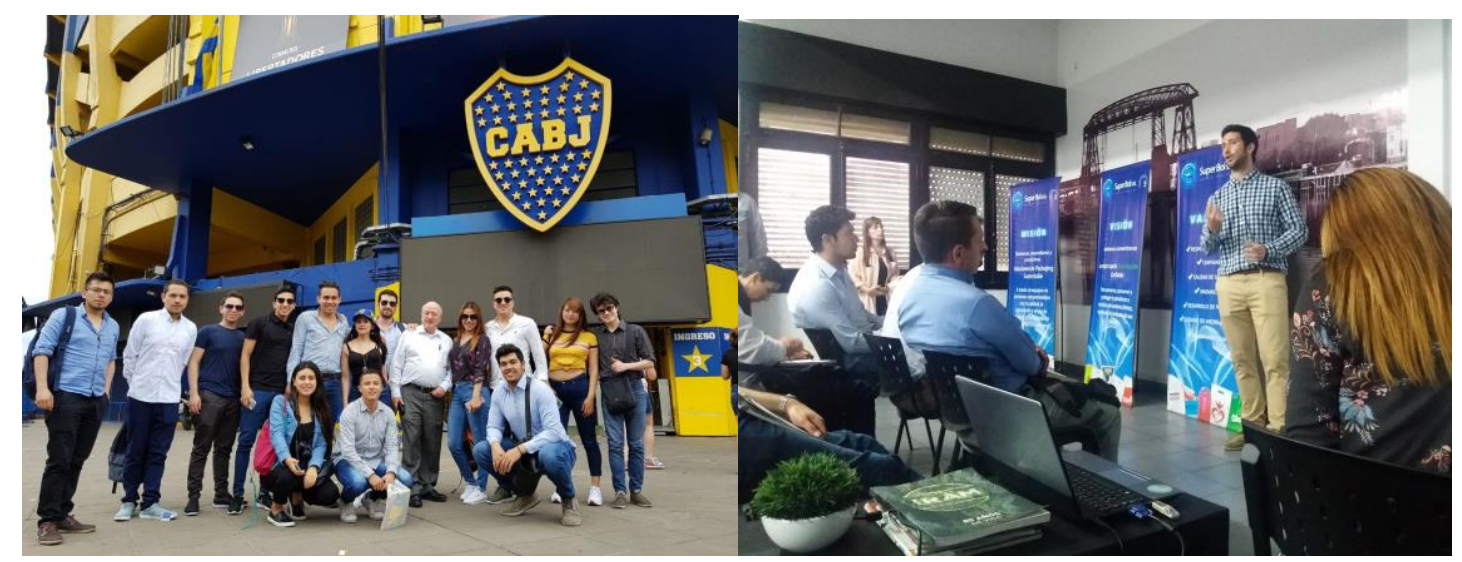

El segundo día, iniciamos la semana visitando una empresa familiar, la cual nos contaba lo difícil que ha sido mantenerse en una economía tan cambiante como la de Argentina, donde desde su fundación ha tenido que enfrentar varias crisis. La empresa se dedica a producir bolsas de plásticos, según lo solicite el cliente. En esta podemos destacar que su mayor cliente es mercado libre, el cual le producen las bolsas de envíos. Al final nos dieron un recorrido por toda la planta de producción y nos explicaron los procesos y el funcionamiento de cada máquina.

Por la tarde, estuvimos en el estadio de la bombonera, el cual tuvimos la oportunidad de ingresar, fuimos al museo del club, donde conocimos la historia del club Boca Juniors, la infraestructura del estadio y tuvimos la posibilidad de ingresar algunas tribunas. 
MARTES, 13 NOV 2018

VISITA PUERTO DE BUENOS AIRES

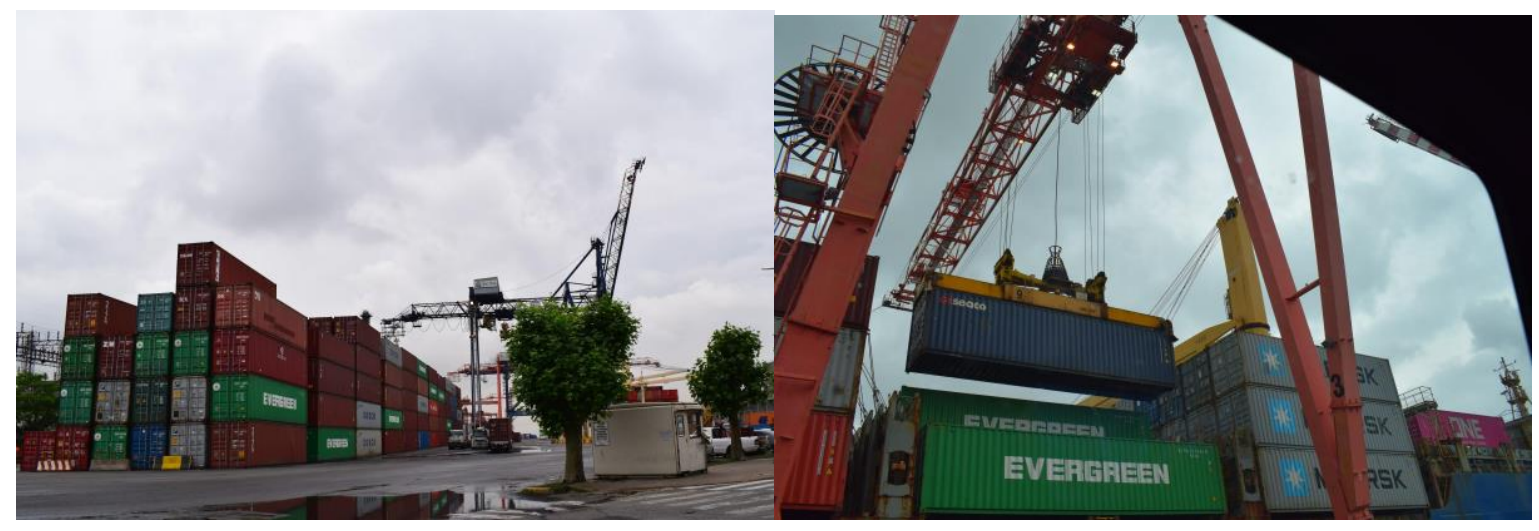

\section{VISITA EMPRESA DE LICOR CAMPARI}

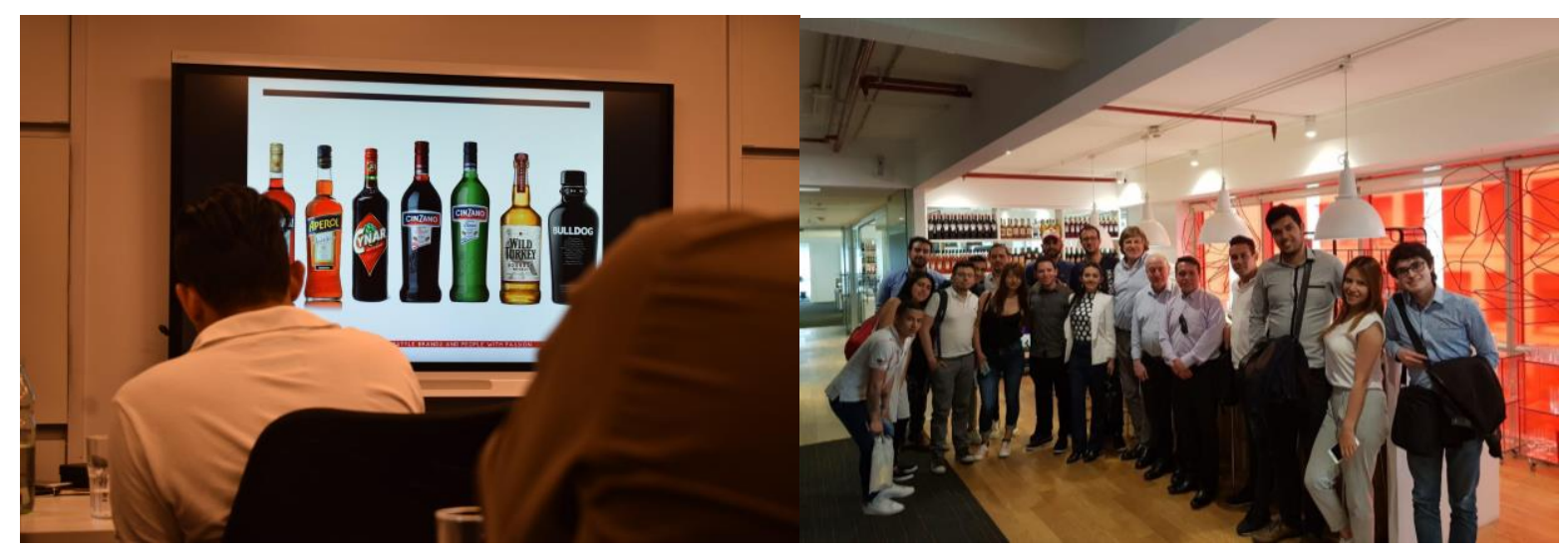

El primer momento del día, recibimos una conferencia en el Puerto de Buenos Aires , el cual nos explicó mediante un mapa la ubicación del puerto, las vías terrestres más importantes que conectaban al puerto con el interior del país y las rutas marítimas, que se utilizaban para el transporte de mercancías, también se resaltó que Colombia y Argentina no poseen una ruta directa para el transporte de contenedores, sino que siempre conectaban con Brasil, además que es uno de los puertos más importantes de Latinoamérica, debido a su gran capacidad de carga superando así el movimiento de más de un millón de contenedores al año, posteriormente nos dirigimos a las zonas de carga y de descarga donde operan 
grandes grúas, para el movimiento de los contenedores, zona de carga a camiones para el transporte terrestre de los contenedores; también el Puerto de Buenos Aires tiene capacidad para el transporte de personas realizando el recorrido hasta Uruguay.

Por otra parte, en la empresa de licores Campari se realizó una conferencia con el gerente de mercadeo, el cual nos explicó que la compañía se encargaba directamente a la fabricación únicamente de bebidas aperitivas (aperitivo (aperire) en latín lo cual significa "abrir el apetito"), esta compañía fue creada en Italia donde existe la fábrica más grande y nos comentó cómo la compañía vende sus productos a través de personas profesionales en la preparación de cócteles; su proceso de marketing es muy importante debido a que ataca directamente las sensaciones que cada cliente puede tener con cada uno de sus productos recalcando la frase "el consumidor no quiere aprender cosas nuevas".

\section{MIÉRCOLES, 14 NOV 2018 VISITA UNIVERSIDAD DE BUENOS AIRES Y TRASLADO A URUGUAY}

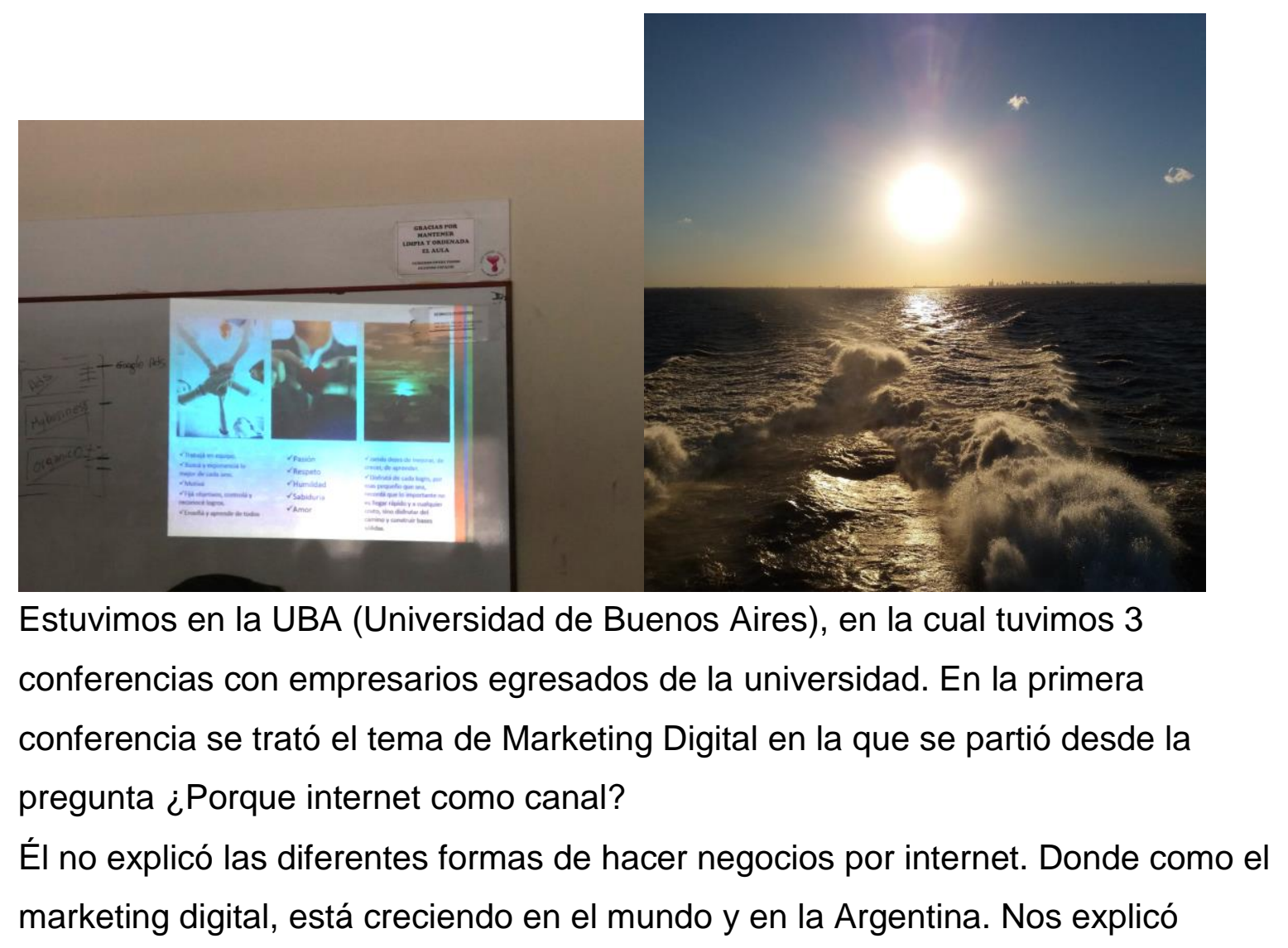


diferentes ideas innovadoras, para pautar en marketing digital y como varias empresas lo utilizan como una ventaja competitiva.

El segundo conferencista, nos habló de un emprendimiento personal. El cual se trataba de una agencia de viajes, donde buscaba que las personas, lograran trabajar en otro país, cambiando de ambiente y buscando ser más productivos y su empresa se encargaba de organizar y buscar los lugares adecuados a un bajo precio.

La última conferencia, también nos habla de un emprendimiento. El cual se trata de un plan de nutrición. Esta empresa les ayuda a las personas a mejorar su calidad de vida cambiando sus hábitos de alimentación y también ofreciéndoles productos saludables que le ayuden con el plan.

Por la tarde abordamos el Ferry, rumbo a Colonia y luego tomamos un bus por dos horas y media que no llevo hasta la ciudad de Montevideo.

\section{JUEVES, 15 NOV 2018}

\section{VISITA AL PUERTO DE URUGUAY Y ALADI}

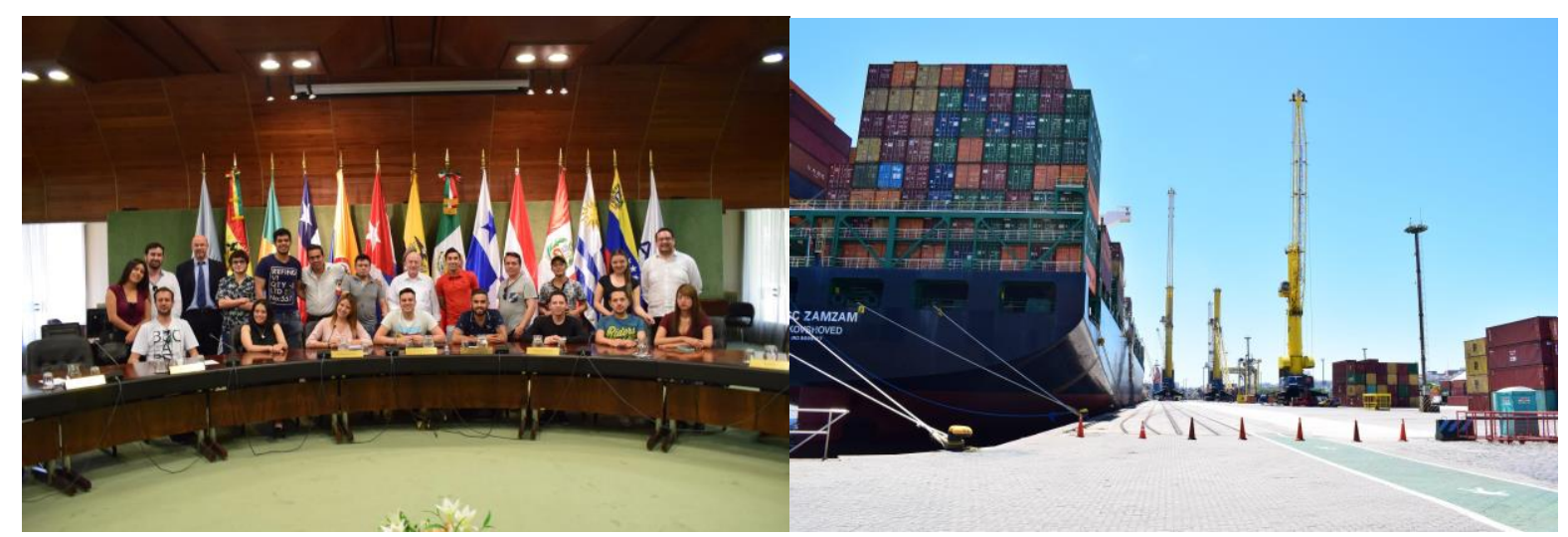

El día jueves muy temprano tuvimos cita en el puerto de Montevideo, el cual se encuentra ubicado sobre el río de La Plata, funciona de forma privada a diferencia del puerto de Buenos Aires el cual es más estatal, pudimos observar las zonas de carga y descarga que posee una capacidad de movimiento de 500.000 contenedores por año. 
En horas de la tarde tuvimos la visita a ALADI, esta es una organización conformada por 13 países: Latinoamérica, Panamá y Cuba, actualmente se encuentra en proceso de adición Nicaragua, esta organización tiene como objetivo quitar las restricciones de comercio y regular el mercado de materias primas buscando un desarrollo en la política comercial, política tecnológica y política industrial.

\section{VIERNES, 16 NOV 2018}

\section{VISITA A ZONAMÉRICA, CITY TOUR POR MONTEVIDEO Y REGRESÓ A BUENOS AIRES}
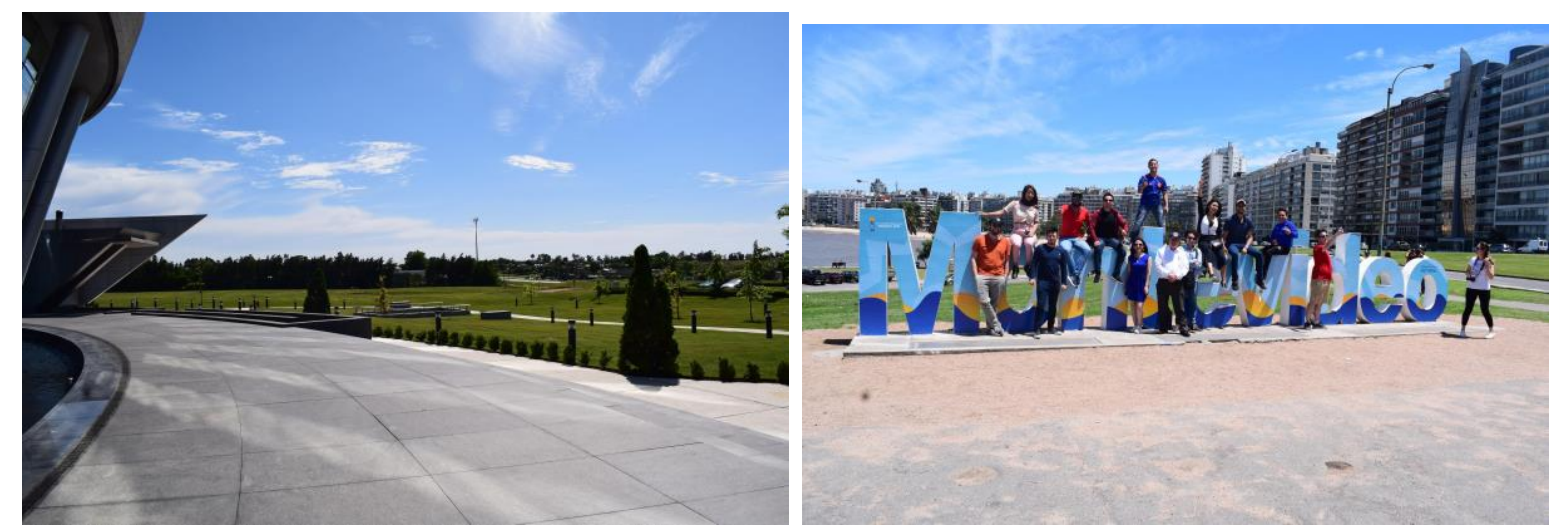

El día viernes tuvimos cita en ZONAMERICA que es una zona franca, un complejo empresarial y tecnológico. Este lugar es un espacio donde las empresas pueden crear su infraestructura de operación, según cada empresa lo necesite.

ZONAMERICA se encarga de ofrecer la mejor infraestructura, tecnología y espacios de satisfacción a los colaboradores del complejo empresarial. Como sabemos las zonas francas son zonas especiales que brinda el gobierno a los empresarios. Uno de los beneficios de esta zona es tener una carga tributaria menor y facilidades para hacer negocios. Sin embargo, estas tienen unas condiciones para los empresarios: Los productos y servicios, no se pueden comercializar en el país debido que están en ventaja con las empresas que no se encuentran en el complejo empresarial. Las empresas deben contratar el 75\%, de personas del país.

Luego de la charla, nos invitaron a desayunar y finalizamos con un recorrido, por todo el complejo empresarial. 


\section{SÁBADO, 17 NOV 2018}

\section{REGRESO A COLOMBIA}

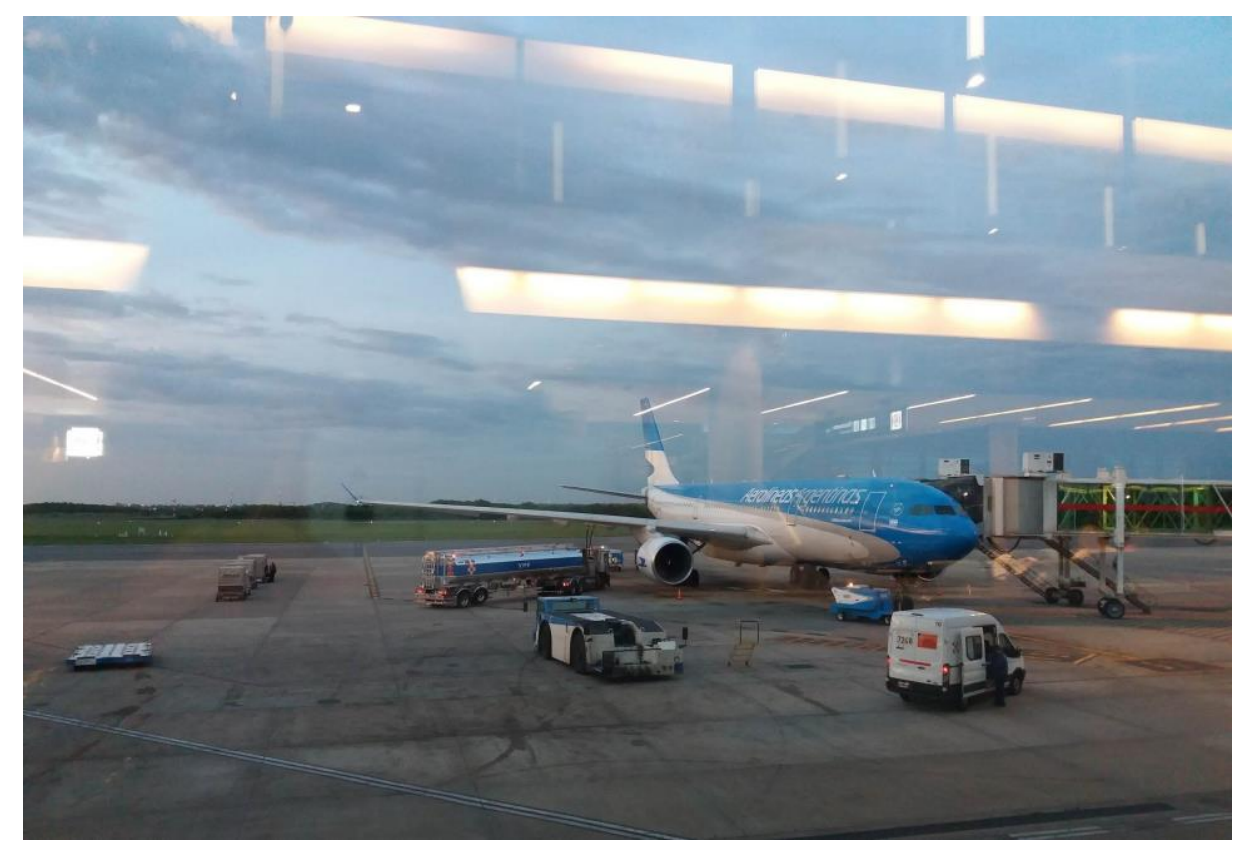

Este día nos recogió el bus a las 3 am y nos dirigimos al aeropuerto, aquí realizamos la entrega de las maletas y nos sentamos a esperar el abordaje que se realizó a las 5:30 am, alrededor de las 10:30am estuvimos aterrizando en Colombia, felices de haber podido vivir esta experiencia que nos enriqueció aún más como profesionales y personas, conocer otra cultura, otras personas, otras costumbres y experiencias de empresas que nacieron de cero, nos inspira como futuros administradores de empresas a emprender y a conocer cómo es la realidad de este entorno empresarial. 